\title{
Mimo Communication System At A Glance
}

\author{
Mrs. Divya, Prashant Khanduri \\ Department of Electronics and Electrical Communication, \\ PEC University of Technology, Chandigarh, India, \\ Department of Electronics and Electrical Communication, \\ PEC University of Technology, Chandigarh, India,
}

\begin{abstract}
Multiple Input Multiple Output (MIMO) communication systems are an important part of modern wireless communication standards. MIMO communication system uses multiple antennas at both transmitting and receiving ends of a communication system. Their use makes it possible to achieve higher capacity of the channel as well as increased data rates order of magnitudes than the conventional Single Input Single Output (SISO) communication systems. This paper presents an overview of a MIMO communication system. Firstly it discusses ergodic and the outage capacity for both schemes when Channel State Information is known and not known at the transmitter of a MIMO channel then it discusses space-time codes for maximization of diversity and hence improving the robustness of the communication link and the most commonly used detection techniques used to extract the correct information from the received signal reducing the Bit Error Rates (BER) to acceptable levels.
\end{abstract}

Keywords: BER, Detection, MIMO, Space-Time codes.

\section{INTRODUCTION}

The MIMO communication system has multiple antennas at both the transmitting and the receiving ends of the channel [1]. Using multiple antennas introduces additional spatial channels on account of which the capacity of the channel increases hence we get increased spectral efficiency without additional bandwidth or transmit power [2],[3]. Because of these properties MIMO communication systems have become an essential part of wireless standards like IEEE 802.11n and IEEE 802.16e WI-MAX. Moreover all upcoming 4G systems will also employ MIMO technology.

The fading channel corrupts the transmitted information symbols and adds noise to the signal through the channel, it is assumed that the signal fades according to the Rayleigh distribution which is applicable when there is no line of sight propagation between the transmitter and the receiver it is a reasonable model for tropospheric and ionospheric signal propagation as well as the effect of heavy build up urban environments on the radio signal. Also the received signal vector $y$ is a linear superposition of separately transmitted information symbols, and this poses a significant challenge to the user to extract the correct information from the received signal. The goal of the MIMO technology is to minimize the BER and simultaneously improve the data rates, this is achieved through the use of spatial multiplexing which aims to increase the data rates and Space Time Codes are used which employ transmit diversity in a optimal and systematic way by adding redundancy to minimize the effects of fading, noise and interference thereby reducing BER [4]. A MIMO system can simultaneously obtain both type of gains, but there is a fundamental tradeoff between how much of each type of gain any signaling scheme can extract [5]. Also the receivers employed must have the capability to extract the correct information from the received signal. The most commonly used detection techniques are Linear Detection, Successive interference cancellation, Maximum-Likelihood Detection (MLD) and Sphere Decoding (SD) [12], [13]. Another major challenge faced is to obtain the channel state information precisely. There are three methods for the channel estimation training based, blind and semi-blind channel estimation.[6]

\section{A. MIMO System Model}

Figure.1. shows the representation of a simple MIMO communication system. Here the transmitted streams go through the $\mathrm{m} \times \mathrm{n}$ channels where $\mathrm{m}$ is the number of transmit antennas and $\mathrm{n}$ is the number of receiving antennas. Assuming two-dimensional elementary constellations throughout, the channel input output relationship is

$$
y=H s+z
$$

Where $s \in \mathbb{C}^{m}, y \in \mathbb{C}^{n}, H \in \mathbb{C}^{n \times m}$ here $s$ is the transmitted signal vector, $y$ is the received signal vector and $\mathrm{H}$ is the $\mathrm{n} \times \mathrm{m}$ complex, possibly random matrix whose entries $h_{i j}$ describe the gains of each transmission path from transmit to receive antenna and $\mathrm{z}$ is the noise vector with vector statistics $z \sim N_{c}\left(0, \sigma^{2} I\right)$. 


\section{B. MIMO Channel}

The channel induces impairments in the communication process therefore a proper characterization of the MIMO channel model is needed to design high performance communication systems.

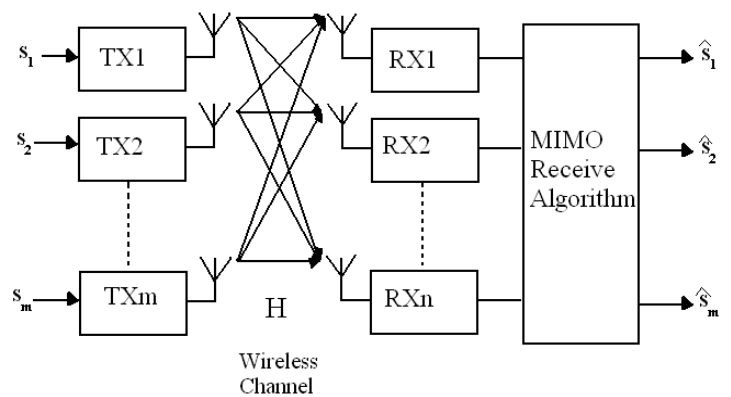

Fig.1 A MIMO Communication system

The channel models can be classified in two ways deterministic and stochastic models. Within the deterministic models we have the recorded impulse response and the ray tracing techniques. On the other hand, stochastic models which aim at reproducing observed phenomena by statistical means can be principally implemented as geometrically-based and correlation-based models[7].

A popular MIMO channel model is the Kronecker model. Its popularity comes from the fact that this correlation based model appear to give more insight in the capacity increase of MIMO channels, since these are based on the channel covariance, establishing a direct link between the channel covariance matrix rank and the channel capacity[8].

\section{MIMO Channel Capacity}

Here the capacity of the MIMO Rayleigh channel first considering that no CSI (equal power allocation) is available at the transmitter and secondly perfect CSI (water filling power allocation) available at the transmitter is given.

In both the cases perfect CSIR is assumed[2][3]. The channel is assumed to be spatially correlated according to a Kronecker model but temporally uncorrelated.

$$
\begin{gathered}
C_{e p}=\sum_{i} \log _{2}\left(1+\frac{S N R}{m} \times \lambda_{i}\right) \\
C_{w f}=\sum_{i} \log _{2}\left(1+P_{l} \times \lambda_{i}\right)
\end{gathered}
$$

Where $C_{e p}$ and $C_{w f}$ denotes the capacity with equal power allocation and capacity with water filling power allocation respectively, SNR is the signal to noise ratio, $\lambda_{i}$ are the Eigen values of the matrix $H \times H^{\prime}$ where $H^{\prime}$ is the Hermitian transpose of matrix $H, m$ are the number of transmit antennas and

$$
P_{l}=\sum_{i}\left(\mu-\lambda_{i}^{-1}\right)
$$

Where $\mu$ is the water filling level. Figure 2 and Figure 3 shows the outage and the ergodic capacities of both equal power allocation and the water filling schemes respectively.

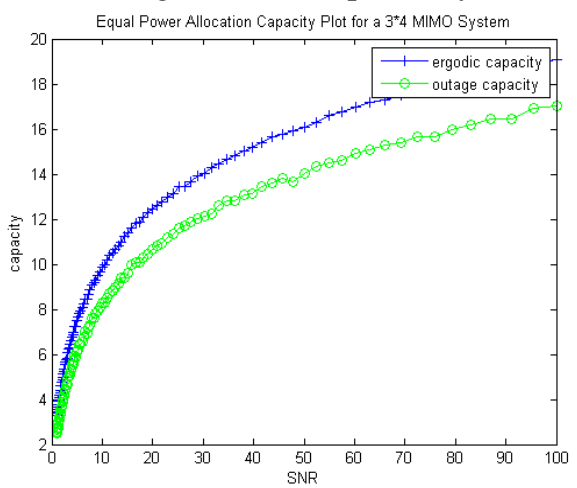

Figure2: Capacity of a 3×4 MIMO Channel with SNR, under equal power allocation scheme. 


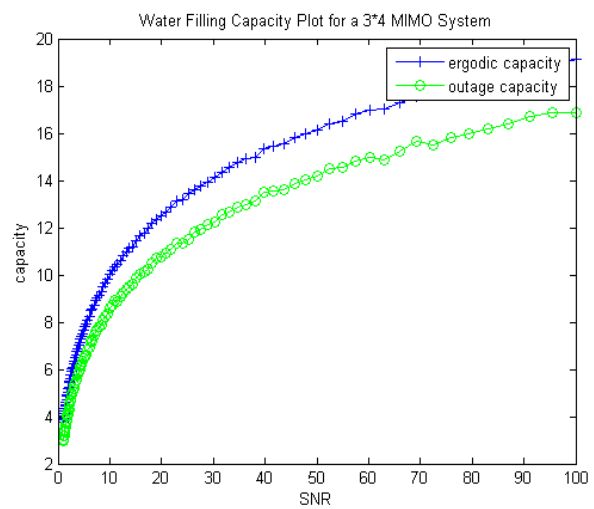

Figure3: Water Filling Capacity of a 3×4 MIMO Channel with SNR.

\section{II.SPACE TIME CODING}

The MIMO system provides two types of gain. The diversity gain and the rate gain. Diversity gain is obtained by space time coding and the rate gain is obtained by spatial multiplexing.

Multiple transmit antennas creates a set of parallel channels that can be used to increase the data rate up to a factor of $\min \{\mathrm{m}, \mathrm{n}\}$ hence generating a rate gain. This is achieved by spatial multiplexing.

The space time codes are designed to provide the transmit diversity, it minimizes the effects of fading and achieve the capacity of the MIMO system by the use of multiple transmit antennas. It increases the robustness of the radio link. Here two main types of space time codes are discussed briefly namely the space-time trellis codes and the space-time block codes.

\section{A. Space time trellis codes(STTC)}

Previously known coding schemes used to expand the bandwidth by introducing redundant bits to the symbols but Trellis codes were introduced to reduce this bandwidth expansion by using a redundant signal set [9].

In a STTC scheme, a stream of data is encoded via $m$ convolution encoders (or via one convolutional encoder with $m$ outputs), where the mapping function is described by a trellis diagram, in order to obtain $m$ modulated streams that are then simultaneously transmitted. Space time codes provide diversity and hence make the system robust. At the receiver, the transmitted frames are decoded by using Viterbi algorithm which implements the Maximum Likelihood estimation. This problem with this detection procedure is that it gives rise to complexity problems with the increase of the trellis code memory length.

\section{B. Space-Time Block Codes (STBC)}

To reduce the decoding complexity of space-time codes, Alamouti [10] proposed a code scheme that uses two transmit antennas and an arbitrary number of receive antennas. This scheme provides full diversity and full data rate for a two antenna system. The key feature of this scheme is the orthogonality between the signal vectors transmitted over the two transmit antennas. This scheme was generalized to an arbitrary number of transmit antennas by applying the theory of orthogonal design [11] and was called STBC.

In general form STBC can be seen as a mapping of $N$ complex symbols $\left\{s_{1}, s_{2} \ldots s_{N}\right\}$ on to a matrix $\boldsymbol{X}$ of dimension $m \times N$

$$
\left\{s_{1}, s_{2} \ldots s_{N}\right\} \rightarrow X
$$

An STBC code matrix $\boldsymbol{X}$ taking on the following form

$$
X=\sum_{n=1}^{N}\left(\operatorname{Re}\left\{s_{n}\right\} A_{n}+j \operatorname{Im}\left\{s_{n}\right\} B_{n}\right)
$$

Here $\left\{s_{1}, s_{2} \ldots s_{N}\right\}$ is a set of symbols to be transmitted, $\left(A_{n}, B_{n}\right)$ are the fixed code matrices of dimension $m \times N$. Each column of the matrix $\boldsymbol{X}$ represents the space time symbol to be transmitted by the $m$ transmitting antennas and matrix $\boldsymbol{X}$ is called the space time codeword. 


\section{III.DETECTION}

The aim here is to detect $s$ in the Maximum Likelihood Sense (ML), which can be written mathematically as

$$
\hat{s}=\arg \min _{s \in S^{m}}\|y-H s\|^{2}
$$

Here the elements of s, say $s_{k}$, belong to a finite alphabet $\mathrm{S}$ of size $|S|$. Hence there are $|S|^{m}$ possible vectors s, $\hat{s}$ is the best estimation for a received signal vector $\mathrm{y}$. The techniques for the detection of spatially multiplexed MIMO signals can be divided into four categories [12][13].

1) Linear Detector: It uses Zero Forcing (ZF) or Minimum Mean Square Criterion (MMSE) to invert the effect of the channel. It shows poor bit error rate (BER) performance.

2) Successive interference cancellation detector: It is only an iterative version of the previous detector. It shows slightly better performance but suffer from error propagation.

3) Maximum-likelihood detector (MLD): It provides optimum maximum likelihood (ML) performance at the expense of complexity.

4) Sphere decoder $(S D)$ : It also provides ML performance while reducing the complexity of the MLD.

\section{QL Decomposition}

Using QL decomposition method, equation (2) can be reformulated as

$$
\hat{s}=\arg \min _{s \in S^{m}}\|\tilde{y}-L s\|^{2}
$$

Where $\tilde{y}=Q^{T} y$

Here $\mathrm{H}$ is decomposed into two matrices $\mathrm{Q}$ and $\mathrm{L}$ so that $\mathrm{H}=\mathrm{QL}$, where $Q \in \mathbb{C}^{n \times m}$ is an orthonormal matrix and $L \in \mathbb{C}^{m \times m}$ is a lower triangular matrix.

We can write the equations in yet another equivalent form as

$$
\min _{\substack{\left\{s_{1}, s_{2} \ldots, s_{m}\right\} \\ s_{k} \in S}}\left\{f_{1}\left(s_{1}\right)+f_{2}\left(s_{1}, s_{2}\right)+\cdots+f_{n}\left(s_{1}, \ldots, s_{m}\right)\right\}
$$

Where $f_{k}\left(s_{1}, \ldots, s_{k}\right) \triangleq\left(\tilde{y}_{k}-\sum_{l=1}^{k} L_{k, l} s_{l}\right)^{2}$

\section{A. Linear Detector: Zero-Forcing (ZF) Detector}

The ZF detector first solves (2) neglecting the constrain $s \in S^{m}$

$$
\begin{gathered}
\tilde{s}=\arg \min _{s \in \mathbb{C}^{m}}\|y-H s\|^{2}=\arg \min _{s \in \mathbb{C}^{m}}\|\tilde{y}-L s\|^{2} \\
=L^{-1} \tilde{y}
\end{gathered}
$$

Here $L^{-1}$ does not need to be computed explicitly after this ZF detector then approximates (2) by projecting each $\widetilde{s_{k}}$ on to the constellation $S$ using

$$
\hat{s}_{k}=\left[\tilde{s}_{k}\right] \triangleq \arg \min _{s_{k} \in S}\left|s_{k}-\tilde{s}_{k}\right|
$$

However ZF works properly only if $\mathrm{H}$ is well conditioned. The reason for this is that the noise is neglected while performing the projection described by equation (6). The correlation between noises in $\tilde{s}_{k}$ can be very significant if $\mathrm{H}$ is not well conditioned.

We can also use MMSE estimate ( $\tilde{s}=E[s / y])$ instead of computing $\tilde{s}$ as in (5). This however improves the performance a little but the fundamental problem is not overcome by it.

\section{B. Successive Interference Cancellation Detector: ZF With Decision Feedback (ZF-DF)}

The ZF-DF approach uses Gaussian Elimination to compute $\tilde{s}$ with only modification to the ZF approach that it projects the symbols on to the constellation $S$ in each step of the Gaussian Elimination rather than afterwards.

a) Detect $s_{1}$ 
Using $\hat{s}_{1}=\arg \min _{s_{1} \in S} f_{1}\left(s_{1}\right)$

$$
=\left[\frac{\tilde{y}_{1}}{L_{1,1}}\right]^{2}
$$

b) Considering $s_{1}$ known $\left(s_{1}=\hat{s}_{1}\right)$, now detecting $s_{2}$ using

$$
\hat{s}_{2}=\arg \min _{s_{2} \in S} f_{2}\left(\hat{s}_{1}, s_{2}\right)
$$

$$
=\left[\frac{\tilde{y}_{2}-\hat{s}_{1} L_{2,1}}{L_{2,2}}\right]^{2}
$$

(c) Now continuing the process for $\mathrm{k}=3,4,5$ and so on to $\mathrm{m}$,

$$
\hat{s}_{k}=\arg \min _{s_{k} \in S} f_{k}\left(\hat{s}_{1}, \ldots, \hat{s}_{k-1}, s_{k}\right)
$$

$$
=\left[\frac{\tilde{y}_{k}-\sum_{l=1}^{k-1} L_{k, l} \hat{s}_{l}}{L_{k, k}}\right]^{2}
$$

The problem with this approach is error propagation, if due to noise interference some incorrect decision is taken in any of the $\mathrm{m}$ steps then this error propagates through to the next levels and is likely to cause errors in subsequent decisions also. In ZF-DF $s_{k}$ is determined in natural order but the detection is not optimal. One can optimize the detection order to minimize the error propagation. However even after optimal ordering of detection the error propagation still limits the performance of the detector.

\section{Maximum Likelihood Detector (MLD) And Sphere Decoding Algorithm}

Maximum Likelihood Detection is optimum detection in the sense of error probability [13]. It solves

$$
\hat{s}=\arg \min _{s \in S^{m}}\|y-H s\|^{2}
$$

Which is same as (2), this equation was reformulated using QL decomposition and was written as (4), this requires an exhaustive search through all lattice points (all possible code words used). However such an exhaustive search is extremely inefficient because it needs to examine $|S|^{m}$ lattice points, this requires very high computational complexity. This computational complexity grows exponentially with the transmission rates and becomes unmanageable for higher data rates [14], therefore the use of MLD is only limited to low data rate applications. To overcome this problem a new approach called sphere decoding has been developed which is discussed next.

To reduce the complexity required to design a MLD while providing the ML performance a promising approach called Sphere Decoding was proposed. The principle of the SD is to search for the closest lattice point to the received signal within a sphere, where each codeword is represented by a lattice point in a lattice field [15][16]. In 2-dimensional field the search can be represented by drawing a circle around the received signal just small enough to include one lattice point and eliminate the search of all the other lattice points that lie outside the circle.

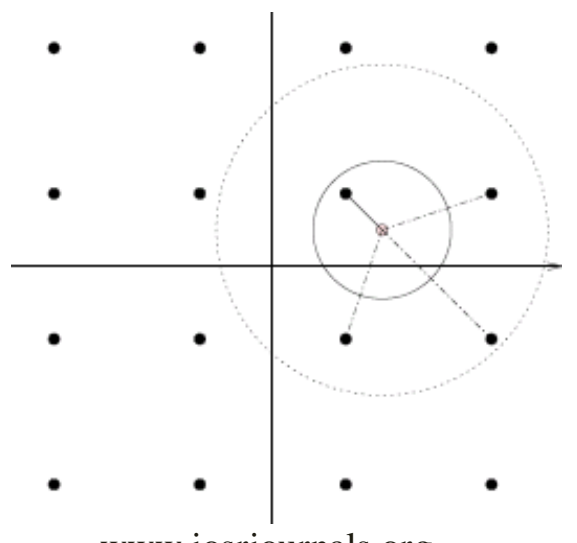

www.iosrjournals.org 
Fig5. 2-D representation of Sphere Decoding algorithm

There are two variants of the sphere decoder algorithm one is hard output which is based on hard decision as a function of the input and the other one is the soft output which is based on soft decision. The soft decoding is more reliable but this reliability comes in expense of the complexity of the decoder.

\section{CONCLUSION}

In this paper we analyzed the capacity of a MIMO channel both when the CSI was known and not known to the transmitter (Figure2 and Figure3). Then overview of two most commonly used Space time coding techniques STTC and STBC was given. Finally the most common detection techniques were briefly discussed with governing equations. The most important of the detection techniques was the Sphere decoding Algorithm as it provides ML detection with low complexity of the detector.

\section{V.REFERENCES}

[1]. A. Paulraj, R. Nabar, and D. Gore, Introduction to Space-Time Wireless Communications. Cambridge Univ. Press, 2003.

[2]. I.E. Telatar, "Capacity of Multi-Antenna Gaussian Channels," Eur. Trans. Telecommun. 10 (6), 1999, pp. 585-595. [3].G. Foschini and M. Gans, "On limits of wireless communications in a fading environment when using multiple antennas" Wireless Personal Communications, vol. 6, no. 3, pp. 311-335, 1998

[4]. Sharma, S.K. Ahmad, S.N. "Performance of MIMO Space-Time Coded Wireless Communication Systems" Conference on Computational Intelligence and Multimedia Applications, 2007

[5]. $\quad$ L. Zheng and D.N.C. Tse. Diversity and multiplexing: A fundamental tradeoff in multiple-antenna channels. IEEE Transactions on Information Theory, 49(5):1073-1096, May 2003.

[6]. Kala Praveen Bagadi , Prof. Susmita Das "MIMO OFDM Channel Estimation Using Pilot Carriers" International Journal of Computer Applications (0975 - 8887) Volume 2 - No.3, May 2010.

[7]. K. Yu and B. Ottersten. Models for mimo propagation channels, a review. Wiley Journal on Wireless Communications and Mobile Computing, 2(6):653-666, November 2002.

[8]. K.I. Pedersen, J.B. Andersen, J.P. Kermoal, and P. Mogensen. A stochastic multiple-input multiple-output radio channel model for evaluation of space-time coding algorithms. In Proceedings of IEEE Vehicular Technology Conference, pages 893-897, September 2000 .

[9] V.Tarokh, N. Seshadri, and A.R. Calderbank. Space-time codes for high data rate wireless communication: Performance criterion and code construction. IEEE Transactions on Information Theory,(2):744-765, March 1998.

[10]. S.M. Alamouti. A simple transmit diversity technique for wireless communications. IEEE Journal on Selected Areas in Communications, 16(8):1451-1458, October 1998.

[11]. V. Tarokh, H. Jafarkhani, and A.R. Calderbank. Space-time block codes from orthogonal designs. IEEE Transactions on Information Theory, 45(5):1456-1467, July 1999.

[12] M. Rupp, M. Guillaud, and S. Das, "On MIMO decoding algorithms for UMTS," in Proc. 35th Asilomar Conference on Signals, Systems and Computers, vol. 2, Monterey, CA, Nov. 2001, pp. 975-979.

[13] M. O. Damen, H. E. Gamal, and G. Caire, "On maximum-likelihood detection and the search for the closest lattice point," IEEE Trans. Inform. Theory, vol. 49, no. 10, pp. 2389-2402, Oct. 2003.

[14] D.Garrett, L. Davis, S. ten Brink, B. Hochwald, and G. Knagge, "Silicon complexity for maximum likelihood MIMO detection using spherical decoding," IEEE J. Solid-State Circuits, vol. 39, pp. 1544-1552, 2004.

[15] E.Agrell, T. Eriksson, A. Vardy, and K. Zeger, "Closest point search in lattices," IEEE Trans. Inform. Theory, vol. 48, pp. 22012214, Aug. 2002

[16] E. Viterbo and J. Boutros, “A universal lattice code decoder for fading channels," IEEE Trans. Inform. Theory, vol. 45, pp. 1639-1642, July 1999 